\title{
COURNOT-NASH EQUILIBRIA IN CONTINUUM GAMES WITH NON-ORDERED PREFERENCES
}

\author{
V.F. MARTINS-DA-ROCHA AND M. TOPUZU
}

\begin{abstract}
In the usual framework of continuum games, we substantially generalize Cournot-Nash existence results (Balder [3, 4, 7]) to games with possibly non-ordered preferences, providing a continuum analogue of the seminal existence results by GaleMas-Colell [12] and Shafer-Sonnenschein [24].

KeYwords. Pure Cournot-Nash equilibria, non-ordered preferences, feeble topology.

JEL Classification. C72.
\end{abstract}

\section{INTRODUCTION}

In recent years attempts were made to extend the seminal equilibrium existence results of Gale-Mas-Colell [12] and Shafer-Sonnenschein [24], formulated for abstract games and economies with a finite set of players, to the framework of continuum economies and games with a measure space of agents. It is proved in Balder [5] that the usual conditions used in the literature for these attempts force the preferred to multifunction to be emptyvalued almost everywhere on the nonatomic part of the measure space, rendering several published extensions pointless. Yet, at the same time, existence results are well-known to hold in continuum games, but only by means of proofs that thoroughly exploit the payoff function structure and its standard-type conditions.

We assert that the modeling of non-ordered preferences provided in Balder [5], BalderYannelis [8], Khan-Papageorgiou [14, 13], Khan-Vohra [15], Kim-Prikry-Yannelis [16], Noguchi $[19,20]$ and Yannelis $[25,26]$ is not relevant to continuum games with externalities. We propose a new modeling of preferences (see Remark 2.1) that encompasses both the modeling of ordered preferences with externalities of Balder $[4,7]$ and the modeling of non-ordered preferences but without externalities of Schmeidler [22], Cornet-TopuzuYildiz [10] and Martins-da-Rocha [17, 18]. Moreover our modeling of non-ordered preferences does not suffer from the serious inconsistency pointed out in Balder [5].

The main result of the paper is the existence of pure Cournot-Nash equilibrium for continuum games with possibly non-ordered preferences. In the framework of ordered preferences (i.e. each agent has a utility/payoff function), the existence of pure CournotNash equilibrium is proved in Balder [4,7] as a corollary of an existence result of mixed

Date: September 20, 2004. 
Cournot-Nash equilibrium by means of Young measure theory. We propose in this paper two independent proofs for our existence result. First (see Section 4.1) we prove that the existence result for games with non-ordered preferences is a simple corollary of the well-known existence result for games with ordered preferences. From a game with nonordered preferences, we define an auxiliary game with payoff functions such that the set of optimal actions for both games coincide. It is then sufficient to apply the existence results in Balder [4, 7]. We also provide (see Section 4.2) a second and independent proof only by means of pure action profiles. This proof involves the feeble topology introduced by Balder [4].

The paper is organized as follows. The model and the main existence result (Theorem 2.1) are presented in Section 2. In Section 3 we provide assumptions on the primitives of the game in order to apply Theorem 2.1. The last section is devoted to the two independent proofs of the main result. For precise definitions of the different continuity concepts for multifunctions used in the paper, we refer to Appendix A.

\section{The MODEL AND THE MAIN RESUlT}

Let $(T, \mathcal{T}, \mu)$ be an abstract finite measure space. The set $T$ is the set of players, which may be a finite set or a continuum such as the unit interval or a mixture of both. For technical reasons we make the following assumption.

Assumption 2.1. The measure space $(T, \mathcal{T}, \mu)$ is complete and separable.

Let $S$ be Hausdorff locally convex topological vector space that is a Suslin space, i.e. $S$ is the continuous image of a Polish space. The space $S$ is the action space. Examples of such spaces include separable Banach spaces, equipped with their norm or weak topology, duals of separable Banach spaces, equipped with their weak star topology, separable Fréchet spaces, such as $\mathcal{C}(\mathbb{R})$, equipped with the compact-open topology, or the space of all bounded, signed measures on a completely regular Suslin space. We denote by $S^{*}$ the topological dual of $S$. For each $t$ in $T$, let $S_{t} \subset S$ denote the action set of player $t$. We denote by $\Sigma$ the multifunction from $T$ into $S$ defined by $\Sigma(t):=S_{t}$.

We let $\bar{T}$ in $\mathcal{T}$ be some fixed measurable subset of players that contains the purely atomic part of $(T, \mathcal{T}, \mu)$. The set $\bar{T}$ is the set of players that will satisfy additional convexity assumptions. We let $\widehat{T}$ denote the set $T \backslash \bar{T}$ and we let $\overline{\mathcal{T}}$ (resp. $\widehat{\mathcal{T}}$ ) be the trace $\sigma$-algebra of $\mathcal{T}$ on $\bar{T}$ (resp. $\widehat{T}$ ). We suppose that the following holds.

Assumption 2.2. For every $t \in T$, the set $S_{t}$ is non-empty and compact, and the graph

$$
D:=\left\{(t, s) \in T \times S: s \in S_{t}\right\}
$$

of the multifunction $\Sigma$ belongs to $\mathcal{T} \otimes \mathcal{B}(S)$. Moreover, for every $t \in \bar{T}$, the set $S_{t}$ is convex. 
We let $\mathcal{D}$ be the trace $\sigma$-algebra of $\mathcal{T} \otimes \mathcal{B}(S)$ on $D$.

An action profile is a function $f: T \rightarrow S$ that is measurable with respect to $\mathcal{T}$ and $\mathcal{B}(S)$ or, equivalently that is scalarly measurable, i.e. for all $s^{*} \in S^{*}$, the scalar functions $t \mapsto\left\langle f(t), s^{*}\right\rangle$ is $\mathcal{T}$-measurable. Let $\mathcal{S}$ denote the set of all action profiles. An action profile $f$ is feasible if $f(t)$ belongs to $S_{t}$ for a.e. $t \in T$. The set of all feasible action profiles is denoted by $\mathcal{S}_{\Sigma}$. Also, let $\overline{\mathcal{S}}_{\Sigma}$ be the set of all restrictions to $\bar{T}$ of functions in $\mathcal{S}_{\Sigma}$; it is only this set that needs to be topologized. We endow $\overline{\mathcal{S}}_{\Sigma}$ with the feeble topology introduced by Balder (see $[4,7]$ ). The feeble topology on $\overline{\mathcal{S}}_{\Sigma}$ is defined as the coarsest topology for which all functionals

$$
J_{g}: f \mapsto \int_{\bar{T}} g(t, f(t)) \mu(d t), \quad g \in \overline{\mathcal{G}}_{L C, \Sigma}
$$

are continuous. Here $\overline{\mathcal{G}}_{L C, \Sigma}$ is the collection of all $\overline{\mathcal{T}} \otimes \mathcal{B}(S)$-measurable functions $g: \bar{T} \times$ $S \rightarrow \mathbb{R}$ for which $g(t,$.$) is linear and continuous on S$ (i.e. belongs to $S^{*}$ ) for every $t \in \bar{T}$ and for which there is an integrable function $\phi_{g}$ in $\mathcal{L}_{\mathbb{R}}^{1}(\bar{T}, \overline{\mathcal{T}}, \mu)$ with $\sup _{s \in S_{t}}|g(t, s)| \leqslant \phi_{g}(t)$ for all $t \in \bar{T}$. The feeble topology can simultaneously subsume the two customary topologies that have been used in the literature on games with a measure space of players (we refer to Balder [4, 7] to precisions and examples). Following Balder [7, Remark 4.3.1] the space $\overline{\mathcal{S}}_{\Sigma}$ is compact, the feeble topology on $\overline{\mathcal{S}}_{\Sigma}$ is semimetrizable and if a sequence $\left(f_{n}\right)$ converges feebly to $f$ in $\overline{\mathcal{S}}_{\Sigma}$, then

$$
f(t) \in \overline{\mathrm{co}} \bigcap_{p \in \mathbb{N}} \mathrm{cl}\left\{f_{n}(t): n \geqslant p\right\}, \quad \text { a.e. } t \in \bar{T} .
$$

Let us now define as the externality of each player $t \in T$ the mapping $d:=(\bar{d}, \hat{d}): \mathcal{S}_{\Sigma} \rightarrow$ $\overline{\mathcal{S}}_{\Sigma} \times \mathbb{R}^{m}$, which is defined by

$$
\bar{d}(f):=\left.f\right|_{\bar{T}} \quad \text { and } \quad \hat{d}(f):=\left(\int_{\widehat{T}} g_{i}(t, f(t)) \mu(d t)\right)_{i=1}^{m} .
$$

Here $\left.f\right|_{\bar{T}} \in \overline{\mathcal{S}}_{\Sigma}$ stands for the restriction to $\bar{T}$ of $f \in \mathcal{S}_{\Sigma}$. Also, for each $i \in\{1, \ldots, m\}$, $g_{i}: D \cap(\widehat{T} \times S) \rightarrow \mathbb{R}$ is a given function that satisfies the following condition.

Assumption 2.3. For each $i \in\{1, \ldots, m\}, g_{i}$ belongs to $\widehat{\mathcal{G}}_{C, \Sigma}$.

Here $\widehat{\mathcal{G}}_{C, \Sigma}$ is the collection of all $\widehat{\mathcal{T}} \otimes \mathcal{B}(S)$-measurable functions $g: \widehat{T} \times S \rightarrow \mathbb{R}$ for which $g(t,$.$) is continuous on S$ for every $t \in \widehat{T}$ and for which there is an integrable function $\phi_{g}$ in $\mathcal{L}_{\mathbb{R}}^{1}(\widehat{T}, \widehat{\mathcal{T}}, \mu)$ with $\sup _{s \in S_{t}}|g(t, s)| \leqslant \phi_{g}(t)$ for all $t \in \widehat{T}$. The externality $d(f)$ depends on the action $f(t)$ of each player $t$ in $\bar{T}$ and depends only on the aggregate $\hat{d}(f)$ over all $\widehat{T}$.

Each player $t \in T$ must choose her actions in accordance with the other players as follows: given the action profile $f \in \mathcal{S}_{\Sigma}$, player $t$ 's socially feasible actions constitute a given subset $A_{t}(d(f)) \subset S_{t}$. 
Until now our model follows almost verbatim the model presented in Balder [4, 7]. Further, we consider a more general framework to model the preference of each player. Every player $t \in T$ has a preferred to multifunction

$$
P_{t}: S_{t} \times \overline{\mathcal{S}}_{\Sigma} \times \mathbb{R}^{m} \rightarrow 2^{S_{t}} .
$$

Given the action profile $\bar{f} \in \overline{\mathcal{S}}_{\Sigma}$, the externality vector $y \in \mathbb{R}^{m}$ and an action $s \in S_{t}$, the set $P_{t}(s, \bar{f}, y)$ represents the set of actions $s^{\prime} \in S_{t}$ that agent $t$ strictly prefers to action $s$.

Example 2.1. In Balder [4, 7], each agent $t$ is endowed with a payoff function $U_{t}$ : $S_{t} \times \overline{\mathcal{S}}_{\Sigma} \times \mathbb{R}^{m} \rightarrow[-\infty,+\infty]$. In that case, $P_{t}$ is defined by

$$
P_{t}(s, \bar{f}, y):=\left\{s^{\prime} \in S_{t}: U_{t}\left(s^{\prime}, \bar{f}, y\right)>U_{t}(s, \bar{f}, y)\right\} .
$$

Remark 2.1. Observe that given an agent $t \in T$, the preferred to multifunction $P_{t}$ is defined on $S_{t} \times \overline{\mathcal{S}}_{\Sigma} \times \mathbb{R}^{m}$. In the literature (see Balder-Yannelis [8], Khan-Papageorgiou [14, 13], Khan-Vohra [15], Kim-Prikry-Yannelis [16], Noguchi [19, 20] and Yannelis [25, 26]), the preferred to multifunction is defined only on $\overline{\mathcal{S}}_{\Sigma} \times \mathbb{R}^{m}$. Moreover it is claimed in Balder [6] that for a game with payoff functions $U_{t}: S_{t} \times \overline{\mathcal{S}}_{\Sigma} \times \mathbb{R}^{m} \rightarrow[-\infty,+\infty]$, the canonical preferred to multifunction for an agent $t \in \bar{T}$ is defined by

$$
P_{t}(\bar{f}, y):=\left\{s \in S_{t}: U_{t}(s, \bar{f}, y)>U_{t}(\bar{f}(t), \bar{f}, y)\right\} .
$$

We claim that this modeling of preferences is not relevant. First because it is proved in Balder [6] that the usual conditions used in the literature for this model force the preferred to multifunctions to be empty valued almost everywhere in the nonatomic part of the measure space of agents. Second because this model does not encompass the literature dealing with games or abstract economies with a measure space of agents but without externalities: in Schmeidler [22], Cornet-Topuzu-Yildiz [10] and Martins-da-Rocha [17, 18] the preferred to multifunction $P_{t}$ is defined on $S_{t}$, hence a natural modeling of preferences to deal with externalities is to consider a preferred to multifunction $P_{t}$ defined on $S_{t} \times \overline{\mathcal{S}}_{\Sigma} \times \mathbb{R}^{m}$. We refer to Balder [7, Section 2.4] for a discussion about the consistency question regarding to our modeling of preferences.

Definition 2.1. For each $t \in T, \bar{f} \in \overline{\mathcal{S}}_{\Sigma}$ and $y \in \mathbb{R}^{m}$, we denote by $M_{t}(\bar{f}, y)$ the set of optimal actions in the socially feasible set $A_{t}(\bar{f}, y)$, i.e.

$$
M_{t}(\bar{f}, y)=\left\{s \in A_{t}(\bar{f}, y): P_{t}(s, \bar{f}, y) \cap A_{t}(\bar{f}, y)=\emptyset\right\} .
$$

Example 2.2. If agent $t$ is endowed with a payoff function $U_{t}: S_{t} \times \overline{\mathcal{S}}_{\Sigma} \times \mathbb{R}^{m} \rightarrow[-\infty,+\infty]$, then $M_{t}(\bar{f}, y)$ coincides with

$$
\operatorname{argmax}_{s \in A_{t}(\bar{f}, y)} U_{t}(s, \bar{f}, y):=\left\{s \in S_{t}: U_{t}(s, \bar{f}, y)=\sup \left\{U_{t}\left(s^{\prime}, \bar{f}, y\right): s^{\prime} \in A_{t}(\bar{f}, y)\right\}\right\} .
$$

We present hereafter the list of assumptions the optimal actions multifunction will be required to satisfy. 
Assumption 2.4 (convexity). For each player $t \in \bar{T}$, the multifunction $M_{t}$ has convex values, i.e. for each $(\bar{f}, y) \in \overline{\mathcal{S}}_{\Sigma} \times \mathbb{R}^{m}$, the set $M_{t}(\bar{f}, y)$ is convex.

Assumption 2.5 (continuity). For each player $t \in T$, the multifunction $(\bar{f}, y) \mapsto M_{t}(\bar{f}, y)$ is upper-semicontinuous with non-empty values.

Assumption 2.6 (measurability). For every $(\bar{f}, y) \in \overline{\mathcal{S}}_{\Sigma} \times \mathbb{R}^{m}$, the multifunction $t \mapsto$ $M_{t}(\bar{f}, y)$ has a measurable graph.

Remark 2.2. Assumption 2.4 is satisfied if for every player $t \in \bar{T}$, for every $(\bar{f}, y) \in$ $\overline{\mathcal{S}}_{\Sigma} \times \mathbb{R}^{m}$ the set $A_{t}(\bar{f}, y)$ and for every action $s \in S_{t}$, the set $\left\{s^{\prime} \in S_{t}: s \notin P_{t}\left(s^{\prime}, \bar{f}, y\right)\right\}$ are convex. In particular, if the preferred to multifunction is defined by a payoff function $U_{t}: S_{t} \times \overline{\mathcal{S}}_{\Sigma} \times \mathbb{R}^{m} \rightarrow[-\infty,+\infty]$ then the last property is satisfied if $s \mapsto U_{t}(s, \bar{f}, y)$ is quasi-concave.

We provide in Section 3 conditions on the primitives $A$ and $P$ of a game such that Assumptions 2.5 and 2.6 are satisfied.

Theorem 2.1. Under Assumptions 2.1 to 2.6, the game $\Gamma:=(T, \Sigma, A, P)$ has a pure Cournot-Nash equilibrium, that is, there exists an action profile $f_{*} \in \mathcal{S}_{\Sigma}$ such that for almost every player $t \in T$,

$$
f_{*}(t) \in A_{t}\left(d\left(f_{*}\right)\right) \quad \text { and } \quad P_{t}\left(f_{*}(t), d\left(f_{*}\right)\right) \cap A_{t}\left(d\left(f_{*}\right)\right)=\emptyset .
$$

Remark 2.3. We propose in Section 4 two approaches to prove Theorem 2.1. In Section 4.1, we consider a game $\Gamma$ satisfying Assumptions 2.1 to 2.6. We define an auxiliary game $\Gamma^{\prime}$ with payoff functions such that optimal action profiles for $\Gamma^{\prime}$ and optimal action profiles for $\Gamma$ coincide. We check that the auxiliary game $\Gamma^{\prime}$ satisfies the set of assumptions needed to apply Theorem 2.2.1 in Balder [7]. We then get the existence of a Cournot-Nash equilibrium for $\Gamma^{\prime}$ which is also a Cournot-Nash equilibrium for the initial game $\Gamma$. Following this approach, our existence for non-ordered preferences appears to be a corollary of the existence results for ordered preferences in Balder [4, 7].

In Section 4.1 we propose a proof purely by means of the feeble topology which is an independent proof of Theorem 2.2.1 in Balder [7]. It was already announced in Balder [4, Section 5] and Balder [7, Remark 4.3.1] that such a proof was possible for ordered preferences. However the proof we propose deals not only with ordered preferences but also with non-ordered preferences.

Remark 2.4. Following the arguments in Balder [7, Section 4.3] we may prove, as a corollary of our pure Cournot-Nash equilibrium existence result (Theorem 2.1), a generalization to non-ordered preferences of the mixed Cournot-Nash equilibrium existence result by Balder [7, Theorem 2.1]. 


\section{Assumptions on PRIMitives}

Let $\Gamma=(T, \Sigma, A, P)$ be a game. We provide in this section conditions on the primitives $A$ and $P$ of the game such that Assumptions 2.5 and 2.6 are satisfied. We first consider a list of assumptions on the multifunction $A$ of socially feasible actions.

Assumption 3.1. For every $(t, \bar{f}, y) \in T \times \overline{\mathcal{S}}_{\Sigma} \times \mathbb{R}^{m}$,

(i) the set $A_{t}(\bar{f}, y)$ is a non-empty and closed subset of $S_{t}$;

(ii) the multifunction $A_{t}: \overline{\mathcal{S}}_{\Sigma} \times \mathbb{R}^{m} \rightarrow 2^{S_{t}}$ is upper-semicontinuous;

(iii) the graph of the multifunction $t \mapsto A_{t}(\bar{f}, y)$ belongs to $\mathcal{T} \otimes \mathcal{B}(S)$.

Remark 3.1. Assumption 3.1 coincides with Assumption 2.2.5 in Balder [7].

We consider now a list of assumptions on the preferred to multifunction $P$.

Assumption 3.2. For every $(t, \bar{f}, y) \in T \times \overline{\mathcal{S}}_{\Sigma} \times \mathbb{R}^{m}$,

(i) for every $s \in S_{t}, s \notin P_{t}(s, \bar{f}, y)$ and one of the two following conditions is satisfied:

a. the multifunction $s \mapsto P_{t}(s, \bar{f}, y)$ is transitive ${ }^{1}$

b. $A_{t}(\bar{f}, y)$ is convex and $s \notin \operatorname{co} P_{t}(s, \bar{f}, y)$ for each $s \in S_{t}$;

(ii) the multifunction $s \mapsto P_{t}(s, \bar{f}, y)$ has open lower-sections;

(iii) the set $\left\{(s, \bar{f}, y) \in S_{t} \times \overline{\mathcal{S}}_{\Sigma} \times \mathbb{R}^{m}: P_{t}(s, \bar{f}, y) \cap A_{t}(\bar{f}, y) \neq \emptyset\right\}$ is open;

(iv) the graph of the multifunction $(t, s) \mapsto P_{t}(s, \bar{f}, y)$ belongs to $\mathcal{T} \otimes \mathcal{B}(S) \otimes \mathcal{B}(S)$.

Remark 3.2. Following Proposition A.1 in Appendix A, Condition (iii) in Assumption 3.2 may be replaced by one of the three following conditions:

(iii.1) the multifunction $P_{t}$ has open lower-sections and open upper-sections, and $A_{t}(\bar{f}, y)$ is the closure of $B_{t}(\bar{f}, y)$ where $B_{t}: \overline{\mathcal{S}}_{\Sigma} \times \mathbb{R}^{m} \rightarrow 2^{S_{t}}$ has open lower-sections;

(iii.2) the graph of the multifunction $P_{t}: S_{t} \times \overline{\mathcal{S}}_{\Sigma} \times \mathbb{R}^{m} \rightarrow 2^{S_{t}}$ is open and the multifunction $A_{t}$ is lower-semicontinuous;

(iii.3) the multifunction $P_{t}$ is lower-semicontinuous and $A_{t}(\bar{f}, y)=S_{t}$.

Corollary 3.1. Let $\Gamma=(T, \Sigma, A, P)$ be a game satisfying Assumptions 2.1 to 2.4, 3.1 and 3.2. Then the game $\Gamma$ has a pure Cournot-Nash equilibrium.

Proof. We can check that under Assumptions 2.1 and 2.2, Assumptions 3.1 and 3.2 imply Assumptions 2.5 and 2.6. Indeed, Assumption 3.1(iii) and Assumption 3.2(iv) imply Assumption 2.6. Assumption 3.1(i) and Assumption 3.2(i-ii) imply that for each $(\bar{f}, y)$ the set $M_{t}(\bar{f}, y)$ is non-empty. Assumption 3.1(ii) and Assumption 3.2(iii) imply that the multifunction $M_{t}$ is upper-semicontinuous.

We provide now a corollary of Corollary 3.1 for games with payoff functions.

\footnotetext{
${ }^{1}$ The multifunction $s \mapsto P_{t}(s, \bar{f}, y)$ is transitive if for each $\left(s, s^{\prime}, s^{\prime \prime}\right)$ in $S_{t}^{3},\left[s^{\prime} \in P_{t}(s, \bar{f}, y)\right.$ and $s^{\prime \prime} \in$ $\left.P_{t}\left(s^{\prime}, \bar{f}, y\right)\right]$ implies $s^{\prime \prime} \in P_{t}(s, \bar{f}, y)$.
} 
Assumption 3.3. For each $t \in T$, agent $t$ is endowed with a payoff function $U_{t}: S_{t} \times$ $\overline{\mathcal{S}}_{\Sigma} \times \mathbb{R}^{m} \rightarrow[-\infty,+\infty]$ such that for every $(t, \bar{f}, y) \in T \times \overline{\mathcal{S}}_{\Sigma} \times \mathbb{R}^{m}$,

(i) the function $U_{t}$ is upper-semicontinuous;

(ii) the function $(t, s) \mapsto U_{t}(s, \bar{f}, y)$ is $\mathcal{D}$-measurable;

(iii) the function $(\bar{f}, y) \mapsto \sup _{s \in A_{t}(\bar{f}, y)} U_{t}(s, \bar{f}, y)$ is lower-semicontinuous on $\overline{\mathcal{S}}_{\Sigma} \times \mathbb{R}^{m}$.

Corollary 3.2. Let $\Gamma=(T, \Sigma, A, P)$ be a game satisfying Assumptions 2.1-2.4, 3.1 and 3.3. Then the game $\Gamma$ has a pure Cournot-Nash equilibrium.

Proof. We can check that under Assumptions 2.1-2.4 and Assumption 3.1, Assumption 3.3 implies Assumption 3.2. Indeed since the preferred to multifunctions are defined by payoff functions, Assumption 3.2(i) is automatically satisfied. Assumption 3.2(ii) follows from Assumption 3.3(i), Assumption 3.2(iv) follows from Assumption 3.3(ii), and Assumption 3.2(iii) follows from Assumptions 3.3(i,iii).

Remark 3.3. Corollary 3.2 coincides with Theorem 2.2.1 in Balder [7]. In particular, Corollary 3.1 generalizes Theorem 2.1 in Balder [4] and Theorem 2.2.1 in Balder [7] to games with possibly non-ordered preferences.

Remark 3.4. In Corollary 3.2, if we replace condition (i) by the following condition

(i') the function $U_{t}$ is continuous;

then under Assumption 3.1, condition (iii) of Assumption 3.3 is automatically satisfied.

\section{Proof of Theorem 2.1}

Let $\Gamma:=(T, \Sigma, A, P)$ be a game satisfying Assumptions 2.1 to 2.4. Since $S$ is Suslin there exists a metric $d$ on $S$ which is weaker than the original topology. Hence on compact subsets of $S$ the original topology and the $d$-topology coincide. Observe that each Borel $\sigma$ algebra corresponding to the $d$-topology coincides with $\mathcal{B}(S)$, because $S$ is Suslin (Corollary 2 of Theorem II.10 of Schwartz [23]).

4.1. Indirect proof. For each player $t \in T$, consider the payoff function $V_{t}: S_{t} \times \overline{\mathcal{S}}_{\Sigma} \times$ $\mathbb{R}^{m} \rightarrow[-\infty,+\infty]$, defined by

$$
\forall(s, \bar{f}, y) \in S_{t} \times \overline{\mathcal{S}}_{\Sigma} \times \mathbb{R}^{m}, \quad V_{t}(s, \bar{f}, y):=-d\left(s, M_{t}(\bar{f}, y)\right)^{2}
$$

We consider now the game $\Gamma^{\prime}:=\left(T, \Sigma, A^{\prime}, P^{\prime}\right)$ which is truly noncooperative, i.e. for each $(\bar{f}, y) \in \overline{\mathcal{S}}_{\Sigma} \times \mathbb{R}^{m}, A_{t}^{\prime}(\bar{f}, y)=S_{t}$. The preferred to multifunction is defined by the payoff function $V$, i.e.

$$
P_{t}^{\prime}(s, \bar{f}, y):=\left\{s^{\prime} \in S_{t}: V_{t}\left(s^{\prime}, \bar{f}, y\right)>V_{t}(s, \bar{f}, y)\right\}
$$

Claim 4.1. For every $t \in T$, the function $V_{t}$ is upper-semicontinuous.

\footnotetext{
${ }^{2}$ If $A$ is a subset of $S$ and $s \in S$ then $d(s, A):=\inf \{d(s, a): a \in A\}$.
} 
Proof. Let $t \in T$ and $c \in \mathbb{R}$, we have to prove that

$$
L:=\left\{(s, \bar{f}, y) \in S_{t} \times \overline{\mathcal{S}}_{\Sigma} \times \mathbb{R}^{m}: d\left(s, M_{t}(\bar{f}, y)\right) \leqslant c\right\}
$$

is closed. Let $\left(s_{n}, \bar{f}_{n}, y_{n}\right)$ be a sequence in $L$ which converges to $(s, \bar{f}, y) \in S_{t} \times \overline{\mathcal{S}}_{\Sigma} \times \mathbb{R}^{m}$. For each $n>0$, there exists $\tilde{s}_{n}$ in $M_{t}\left(\bar{f}_{n}, y_{n}\right)$ such that $d\left(s_{n}, \tilde{s}_{n}\right) \leqslant c+1 / n$. Since $S_{t}$ is compact, passing to a subsequence if necessary, we can suppose that $\left(\tilde{s}_{n}\right)$ is convergent to $\tilde{s}$ in $S_{t}$. From Assumption 2.5, $\tilde{s}$ belongs to $M_{t}(\bar{f}, y)$, hence

$$
d\left(s, M_{t}(\bar{f}, y)\right) \leqslant d(s, \tilde{s})=\lim _{n} d\left(s_{n}, \tilde{s}_{n}\right) \leqslant c .
$$

Claim 4.2. For each $(\bar{f}, y) \in \overline{\mathcal{S}}_{\Sigma} \times \mathbb{R}^{m}$, the multifunction $(t, s) \mapsto V_{t}(s, \bar{f}, y)$ defined on $D$ is $\mathcal{D}$-measurable.

Proof. Let $(\bar{f}, y) \in \overline{\mathcal{S}}_{\Sigma} \times \mathbb{R}^{m}$, we let $F: T \rightarrow 2^{S}$ be the multifunction defined by $F(t):=$ $M_{t}(\bar{f}, y)$. From Assumption 2.6 the multifunction $F$ has a measurable graph. From Castaing-Valadier [9, Theorem III.22] (see also Sainte Beuve [21]), there exists a sequence $\left(\sigma_{n}\right)$ of measurable functions $\sigma_{n}: T \rightarrow S$ such that $\left(\sigma_{n}(t)\right)$ is dense in $F(t)$ for every $t \in T$. It follows that for each $s \in S$,

$$
d(s, F(t))=\inf \left\{d\left(s, \sigma_{n}(t)\right): n \in \mathbb{N}\right\} .
$$

Hence for each $s \in S$ the function $t \mapsto d(s, F(t))$ is measurable, and for each $t \in T$, the function $s \mapsto d(s, F(t))$ is continuous. Applying Lemma III.14 in Castaing-Valadier [9], we get that $(t, s) \mapsto d(s, F(t))$ is $\mathcal{T} \otimes \mathcal{B}(S)$-measurable. Since $F(t)$ is a subset of $S_{t}$, it follows that $(t, s) \mapsto d(s, F(t))$ is $\mathcal{D}$-measurable.

Claim 4.3. For every $t \in T$, for every $(\bar{f}, y) \in \overline{\mathcal{S}}_{\Sigma} \times \mathbb{R}^{m}$,

$$
\operatorname{argmax}_{s \in S_{t}} V_{t}(s, \bar{f}, y)=M_{t}(\bar{f}, y) \text {. }
$$

Proof. Let $t \in T$ and $(\bar{f}, y) \in \overline{\mathcal{S}}_{\Sigma} \times \mathbb{R}^{m}$. From Assumption 2.5, the set $M_{t}(\bar{f}, y)$ is nonempty, hence there exists $\sigma \in S_{t}$ such that $V_{t}(\sigma, \bar{f}, y)=0$. Now since $V_{t}(s, \bar{f}, y) \leqslant 0$ for each $s \in S_{t}$, we have that

$$
\operatorname{argmax}_{s \in S_{t}} V_{t}(s, \bar{f}, y)=\left\{s \in S_{t}: d\left(s, M_{t}(\bar{f}, y)\right)=0\right\} .
$$

From Assumption 2.5, $M_{t}(\bar{f}, y)$ is closed, hence the claim follows.

We claim that the game $\Gamma^{\prime}$ satisfies Assumptions 2.2.5 to 2.2.7 in Balder [7]. Indeed, the game $\Gamma^{\prime}$ is truly noncooperative, in the sense that for each $(\bar{f}, y) \in \overline{\mathcal{S}}_{\Sigma} \times \mathbb{R}^{m}$, for every $t \in T, A_{t}^{\prime}(\bar{f}, y)=S_{t}$. Hence Assumption 2.2.5 in [7] is trivially satisfied. From Claims 4.1 and 4.2, Assumption 2.2.6 in [7] is satisfied. Observe that for every $t \in T$, the function

$$
(\bar{f}, y) \longmapsto \sup \left\{V_{t}(s, \bar{f}, y): s \in S_{t}\right\}
$$


is identical to zero, so Assumption 2.2.7.(i) in [7] is trivially satisfied. Assumption 2.2.7.(ii) in [7] follows from Claim 4.3 and Assumption 2.4.

Now we can apply Theorem 2.2.1 in Balder [7] to get the existence of a Cournot-Nash equilibrium $f_{*}$ for the game $\Gamma^{\prime}$. Obviously $f_{*}$ is also a Cournot-Nash equilibrium for the game $\Gamma$.

4.2. Direct proof. For each $(\bar{f}, y) \in \overline{\mathcal{S}}_{\Sigma} \times \mathbb{R}^{m}$ we let $\overline{\mathcal{S}}_{M(\bar{f}, y)}$ denote the set of all functions $\bar{g} \in \overline{\mathcal{S}}_{\Sigma}$ such that $\bar{g}(t) \in M_{t}(\bar{f}, y)$ for a.e. $t \in \bar{T}$.

Claim 4.4. The multifunction $(\bar{f}, y) \longmapsto \overline{\mathcal{S}}_{M(\bar{f}, y)}$ is upper-semicontinuous with compact convex and non-empty values.

Let $Y$ be the subset of $\mathbb{R}^{m}$ defined by

$$
Y:=\left\{y \in \mathbb{R}^{m}: \exists \hat{f} \in \widehat{\mathcal{S}}_{\Sigma}, \quad \forall j \in\{1, \ldots, m\}, \quad y_{j}=\int_{\widehat{T}} g_{j}(t, \widehat{f}(t)) \mu(d t)\right\},
$$

where $\widehat{\mathcal{S}}_{\Sigma}$ is the set of all restrictions to $\widehat{T}$ of functions in $\mathcal{S}_{\Sigma}$.

Claim 4.5. The set $Y$ is compact convex and non-empty.

For each $(\bar{f}, y) \in \overline{\mathcal{S}}_{\Sigma} \times \mathbb{R}^{m}$ let $\mathcal{Y}(\bar{f}, y)$ be the subset of $Y$ defined by

$$
\mathcal{Y}(\bar{f}, y):=\left\{z \in \mathbb{R}^{m}: \exists \hat{h} \in \widehat{\mathcal{S}}_{M(\bar{f}, y)}, \quad \forall j \in\{1, \ldots, m\}, \quad z_{j}=\int_{\widehat{T}} g_{j}(t, \widehat{h}(t)) \mu(d t)\right\},
$$

where $\widehat{\mathcal{S}}_{M(\bar{f}, y)}$ is the set of all functions $\hat{g} \in \widehat{\mathcal{S}}_{\Sigma}$ such that $\hat{g}(t) \in M_{t}(\bar{f}, y)$ for a.e. $t \in \widehat{T}$.

Claim 4.6. The multifunction $(\bar{f}, y) \longmapsto \mathcal{Y}(\bar{f}, y)$ is upper-semicontinuous with compact convex and non-empty values.

Following Claims 4.4-4.6, the multifunction

$$
(\bar{f}, y) \longmapsto \overline{\mathcal{S}}_{M(\bar{f}, y)} \times \mathcal{Y}(\bar{f}, y)
$$

defined on the non-empty compact convex $\overline{\mathcal{S}}_{\Sigma} \times Y$ is upper-semicontinuous with nonempty compact convex values. Applying a non-Hausdorff version of Kakutani's fixed-point theorem (see Balder [4, Theorem A.2]), there exists $\left(\bar{f}_{*}, y_{*}\right) \in \overline{\mathcal{S}}_{\Sigma} \times Y$ such that

$$
\bar{f}_{*} \in \overline{\mathcal{S}}_{M\left(\bar{f}_{*}, y_{*}\right)} \quad \text { and } \quad y_{*} \in \mathcal{Y}\left(\bar{f}_{*}, y_{*}\right) .
$$

Hence there exists $\hat{f}_{*} \in \widehat{\mathcal{S}}_{M\left(\bar{f}_{*}, y_{*}\right)}$ such that $y_{*}=\hat{d}\left(\hat{f}_{*}\right)$. Let $f_{*}$ be the function defined by

$$
f_{*}(t)=\left\{\begin{array}{l}
\hat{f}_{*}(t) \quad \text { if } \quad t \in \widehat{T} \\
\bar{f}_{*}(t) \text { if } t \in \bar{T} .
\end{array}\right.
$$

The function $f_{*}$ belongs to $\mathcal{S}_{\Sigma}$ and $\left(\bar{f}_{*}, y_{*}\right)=d\left(f_{*}\right)$. Following $(4.1), f_{*}(t) \in M_{t}\left(d\left(f_{*}\right)\right)$ for a.e. $t \in T$, i.e. $f_{*}$ is a Cournot-Nash equilibrium of $\Gamma$. 


\section{Appendix A. Continuous multifunctions}

Let $X$ and $Y$ two semimetrizable topological vector spaces and $F$ a multifunction from $X$ to $Y$.

Definition A.1. The multifunction $F$ is said: to be upper-semicontinuous if for each open set $V \subset Y$ the set $\{x \in X: F(x) \subset V\}$ is open; to have a closed graph if the set $\{(x, y) \in X \times Y: y \in F(x)\}$ is closed.

We recall the following well-known equivalence result (see e.g. Florenzano [11, Appendix A]). If there exists a compact set $K \subset Y$ such that for each $x \in X, F(x) \subset K$ then the multifunction $F$ is upper-semicontinuous if and only if it has a closed graph.

Definition A.2. The multifunction $F$ is said: to have an open graph if $\{(x, y) \in X \times$ $Y: y \in F(x)\}$ is open; to have open lower-sections if for each $y \in Y$, the set $\{x \in$ $X: F(x) \ni y\}$ is open; to have open upper-sections if for each $x \in Y$, the set $F(x)$ is open; and to be lower-semicontinuous if for each non-empty open set $V \subset Y$ the set $\{x \in X: F(x) \cap V \neq \emptyset\}$ is open.

Proposition A.1. Let $S$ and $\Delta$ be Hausdorff topological spaces and consider a multifunction $P$ from $S \times \Delta$ to $S$ and $A$ from $\Delta$ to $S$. Consider the following conditions:

(iii.1) the multifunction $P$ has open lower-sections and open upper-sections and for each $\delta \in \Delta, A(\delta)$ is the closure of $B(\delta)$ where the multifunction $B: \Delta \rightarrow S$ has open lower-sections;

(iii.2) the graph of the multifunction $P: S \times \Delta \rightarrow S$ is open and the multifunction $A$ is lower-semicontinuous;

(iii.3) the multifunction $P$ is lower-semicontinuous and $A(\delta)=S$ for each $\delta \in \Delta$.

Then each one of the above conditions imply the following one:

(iii) the set $\{(s, \delta) \in S \times \Delta: P(s, \delta) \cap A(\delta) \neq \emptyset\}$ is open.

Proof of: (iii1) implies (iii). For each $(s, \delta) \in S \times \Delta, P(s, \delta)$ is open hence $P(s, \delta) \cap A(\delta) \neq$ $\emptyset$ if and only if $P(s, \delta) \cap B(\delta) \neq \emptyset$. Let $(s, \delta) \in S \times \Delta$ such that $P(s, \delta) \cap B(\delta) \neq \emptyset$ and let $\sigma \in S$ be such that $\sigma \in P(s, \delta) \cap B(\delta)$. Since $P$ has open lower-sections there exist open sets $U$ and $V$ in $S$ and $\Delta$ such that $(s, \delta) \in U \times V$ and for each $\left(s^{\prime}, \delta^{\prime}\right) \in U \times V$, $P\left(s^{\prime}, \delta^{\prime}\right) \ni \sigma$. Since $B$ has open lower-sections there exists an open set $W$ in $\Delta$ such that $\delta \in W$ and for each $\delta^{\prime} \in W, B\left(\delta^{\prime}\right) \ni \sigma$. It then follows that $(s, \delta) \in U \times(V \cap W)$ and for each $\left(s^{\prime}, \delta^{\prime}\right) \in U \times(V \cap W), P\left(s^{\prime}, \delta^{\prime}\right) \cap B\left(\delta^{\prime}\right) \neq \emptyset$.

Proof of: (iii2) implies (iii). Let $(s, \delta) \in S \times \Delta$ such that $P(s, \delta) \cap A(\delta) \neq \emptyset$. Let $\sigma \in S$ be such that $\sigma \in P(s, \delta) \cap A(\delta)$. Since $P$ has an open graph, there exists two open sets $U, V$ in $S$ and an open set $W$ in $\Delta$ such that $(s, \delta, \sigma) \in U \times W \times V$ and for each $\left(s^{\prime}, \delta^{\prime}, \sigma^{\prime}\right) \in U \times W \times V, P\left(s^{\prime}, \delta^{\prime}\right) \cap A\left(\delta^{\prime}\right) \ni \sigma^{\prime}$. The multifunction $A$ is lower semi-continuous hence the set $\left\{\delta^{\prime} \in \Delta: A\left(\delta^{\prime}\right) \cap V \neq \emptyset\right\}$ is open. Therefore there exists an open set $W^{\prime}$ 
in $\Delta$ such that $\delta \in W^{\prime}$ and for each $\delta^{\prime} \in W^{\prime}, A\left(\delta^{\prime}\right) \cap V \neq \emptyset$. It then follows that $(s, \delta) \in U \times\left(W \cap W^{\prime}\right)$ and for each $\left(s^{\prime}, \delta^{\prime}\right) \in U \times\left(W \cap W^{\prime}\right), P\left(s^{\prime}, \delta^{\prime}\right) \cap A\left(\delta^{\prime}\right) \neq \emptyset$.

Proof of: (iii3) implies (iii). For each $(s, \delta) \in S \times \Delta, P(s, \delta) \cap A(\delta) \neq \emptyset$ if and only if $P(s, \delta) \cap S \neq \emptyset$. The set $S$ is open, hence (iii) follows from the lower semi-continuity of the multifunction $P$.

\section{Appendix B. Proof of Claim 4.4}

We first prove that for each $(\bar{f}, y)$, the set $\overline{\mathcal{S}}_{M(\bar{f}, y)}$ is non-empty and convex. Fix $(\bar{f}, y)$, from Assumption 2.6 the multifunction $t \mapsto M_{t}(\bar{f}, y)$ has a measurable graph. By applying the von Neumann-Aumann measurable selection theorem (Theorem III.22 of CastaingValadier [9]) there exists a measurable selection of $t \mapsto M_{t}(\bar{f}, y)$. This implies that the set $\overline{\mathcal{S}}_{M(\bar{f}, y)}$ is non-empty. The convexity follows from Assumption 2.4.

We now prove that the multifunction $(\bar{f}, y) \mapsto \overline{\mathcal{S}}_{M(\bar{f}, y)}$ is upper-semicontinuous. For each $(\bar{f}, y)$, the set $\overline{\mathcal{S}}_{M(\bar{f}, y)}$ is a subset of the compact set $\overline{\mathcal{S}}_{\Sigma}$. Hence to prove the uppersemicontinuity, we consider a sequence $\left(\bar{f}_{n}, y_{n}, \bar{g}_{n}\right)$ in $\overline{\mathcal{S}}_{\Sigma} \times \mathbb{R}^{m} \times \overline{\mathcal{S}}_{\Sigma}$ converging to $(\bar{f}, y, \bar{g})$ in $\overline{\mathcal{S}}_{\Sigma} \times \mathbb{R}^{m} \times \overline{\mathcal{S}}_{\Sigma}$ such that

$$
\forall n \in \mathbb{N}, \quad \bar{g}_{n} \in \overline{\mathcal{S}}_{M\left(\bar{f}_{n}, y_{n}\right)} .
$$

We have to prove that $\bar{g}$ belongs to $\overline{\mathcal{S}}_{M(\bar{f}, y)}$. From $(2.1)$

$$
\bar{g}(t) \in \overline{\mathrm{co}} \bigcap_{p \in \mathbb{N}} \operatorname{cl}\left\{f_{n}(t): n \geqslant p\right\} \quad \text { a.e. } t \in \bar{T} .
$$

From Assumption 2.5 the multifunction $M_{t}(.,$.$) is upper-semicontinuous and then$

$$
\bigcap_{p \in \mathbb{N}} \operatorname{cl}\left\{f_{n}(t): n \geqslant p\right\} \subset M_{t}(\bar{f}, y) \quad \text { a.e. } t \in \bar{T} \text {. }
$$

From Assumptions 2.4 and 2.5, the set $M_{t}(\bar{f}, y)$ is closed convex, thus

$$
\bar{g}(t) \in \overline{\mathrm{co}} \bigcap_{p \in \mathbb{N}} \operatorname{cl}\left\{f_{n}(t): n \geqslant p\right\} \subset M_{t}(\bar{f}, y) \quad \text { a.e. } t \in \bar{T} \text {. }
$$

\section{Appendix C. Proof of Claim 4.5}

We first prove that the set $Y$ is non-empty and convex. From Assumption 2.2 the multifunction $t \mapsto S_{t}$ has a measurable graph. By applying the von Neumann-Aumann measurable selection theorem (Theorem III.22 of Castaing-Valadier [9]) there exists a measurable selection of $t \mapsto S_{t}$. This implies that the set $\widehat{\mathcal{S}}_{\Sigma}$ is non-empty and thus $Y$ is non-empty. The convexity follows from the Extended Lyapunov Theorem in Balder [2]. 
We now prove that $Y$ is a compact set. From Assumption 2.3, the set $Y$ is bounded. Consider now a sequence $\left(y^{n}\right)$ in $Y$ converging to $y \in \mathbb{R}^{m}$. There exists a sequence $\left(\hat{f}_{n}\right)$ in $\widehat{\mathcal{S}}_{\Sigma}$ such that

$$
\forall j \in\{1, \ldots, m\}, \quad y_{j}^{n}=\int_{\widehat{T}} g_{j}\left(t, \hat{f}_{n}(t)\right) \mu(d t) .
$$

For each $n \in \mathbb{N}$, denote by $\varphi_{n}(t)$ the vector $\left(g_{j}\left(t, \hat{f}_{n}(t)\right)\right)$ in $\mathbb{R}^{m}$. From the multivalued Fatou's Lemma by Artstein [1], there exists an integrable mapping $\varphi: \widehat{T} \rightarrow \mathbb{R}^{m}$ such that

$$
y=\int_{\widehat{T}} \varphi(t) \mu(d t) \quad \text { and } \quad \varphi(t) \in \overline{\operatorname{co}} \bigcap_{p \in \mathbb{N}} \operatorname{cl}\left\{\varphi_{n}(t): n \geqslant p\right\} \quad \text { a.e. } t \in \widehat{T} .
$$

For each $t \in \widehat{T}$, the set $S_{t}$ is compact and the mapping $g_{j}(t,$.$) is continuous. It then$ follows that for almost every $t \in \widehat{T}$ there exists $s_{t} \in S_{t}$ such that $\varphi_{j}(t)=g_{j}\left(t, s_{t}\right)$ for every $j \in\{1, \ldots, m\}$. Let $\Sigma^{\varphi}: \widehat{T} \rightarrow S$ be the multifunction defined by

$$
\forall t \in \widehat{T}, \quad \Sigma^{\varphi}(t)=\left\{s \in \Sigma(t): g_{j}(t, s)=\varphi_{j}(t) \quad \forall j \in\{1, \ldots, m\}\right\} .
$$

The multifunction $\Sigma^{\varphi}$ has a measurable graph with non-empty values. By applying the von Neumann-Aumann measurable selection theorem (Theorem III.22 of CastaingValadier [9]) there exists a measurable selection $\hat{f}$ of $\Sigma^{\varphi}$. In particular

$$
y_{j}=\int_{\widehat{T}} g_{j}(t, \hat{f}(t)) \mu(d t) \quad \forall j \in\{1, \ldots, m\},
$$

which yields that $y \in Y$.

\section{Appendix D. Proof of Claim 4.6}

We first prove that for each $(\bar{f}, y)$, the set $\mathcal{Y}(\bar{f}, y)$ is non-empty and convex. Fix $(\bar{f}, y)$, from Assumption 2.6 the multifunction $t \mapsto M_{t}(\bar{f}, y)$ has a measurable graph. By applying the von Neumann-Aumann measurable selection theorem (Theorem III.22 of CastaingValadier [9]) there exists a measurable selection of $t \mapsto M_{t}(\bar{f}, y)$. This implies that the set $\widehat{\mathcal{S}}_{M(\bar{f}, y)}$ is non-empty. The convexity follows from the Extended Lyapunov Theorem in Balder [2].

We now prove that the multifunction $(\bar{f}, y) \mapsto \mathcal{Y}(\bar{f}, y)$ is upper-semicontinuous. For each $(\bar{f}, y)$, the set $\mathcal{Y}(\bar{f}, y)$ is a subset of the compact set $Y$. Hence to prove the uppersemicontinuity, we consider a sequence $\left(\bar{f}_{n}, y_{n}, z^{n}\right)$ in $\overline{\mathcal{S}}_{\Sigma} \times \mathbb{R}^{m} \times Y$ converging to $(\bar{f}, y, z)$ in $\overline{\mathcal{S}}_{\Sigma} \times \mathbb{R}^{m} \times Y$ such that

$$
\forall n \in \mathbb{N}, \quad z^{n} \in \mathcal{Y}\left(\bar{f}_{n}, y_{n}\right) .
$$

We have to prove that $z$ belongs to $\mathcal{Y}(\bar{f}, y)$. There exists a sequence $\left(\hat{h}_{n}\right)$ in $\widehat{\mathcal{S}}_{M\left(\bar{f}_{n}, y_{n}\right)}$ such that

$$
\forall j \in\{1, \ldots, m\}, \quad z_{j}^{n}=\int_{\widehat{T}} g_{j}\left(t, \hat{h}_{n}(t)\right) \mu(d t) .
$$


For each $n \in \mathbb{N}$, denote by $\varphi_{n}(t)$ the vector $\left(g_{j}\left(t, \hat{h}_{n}(t)\right)\right)$ in $\mathbb{R}^{m}$. From the multivalued Fatou's Lemma by Artstein [1], there exists an integrable mapping $\varphi: \widehat{T} \rightarrow \mathbb{R}^{m}$ such that

$$
z=\int_{\widehat{T}} \varphi(t) \mu(d t) \quad \text { and } \quad \varphi(t) \in \overline{\mathrm{co}} \bigcap_{p \in \mathbb{N}} \operatorname{cl}\left\{\varphi_{n}(t): n \geqslant p\right\} \quad \text { a.e. } t \in \widehat{T} .
$$

For each $t \in \widehat{T}$, the multifunction $M_{t}(.,$.$) is upper-semicontinuous and the mapping g_{j}(t,$. is continuous. It then follows that for almost every $t \in \widehat{T}$ there exists $s_{t} \in M_{t}(\bar{f}, y)$ such that $\varphi_{j}(t)=g_{j}\left(t, s_{t}\right)$ for every $j \in\{1, \ldots, m\}$. Let $\Sigma^{M}: \widehat{T} \rightarrow S$ be the multifunction defined by

$$
\forall t \in \widehat{T}, \quad \Sigma^{M}(t)=\left\{s \in M_{t}(\bar{f}, y): g_{j}(t, s)=\varphi_{j}(t) \quad \forall j \in\{1, \ldots, m\}\right\} .
$$

From Assumption 2.6 the multifunction $\Sigma^{M}$ has a measurable graph with non-empty values. By applying the von Neumann-Aumann measurable selection theorem (Theorem III.22 of Castaing-Valadier [9]) there exists a measurable selection $\hat{h}$ of $\Sigma^{M}$. In particular

$$
z_{j}=\int_{\widehat{T}} h_{j}(t, \hat{f}(t)) \mu(d t) \quad \forall j \in\{1, \ldots, m\},
$$

which yields that $z \in \mathcal{Y}(\bar{f}, y)$.

\section{REFERENCES}

1. Z. Artstein, A note on Fatou's lemma in several dimensions, J. Math. Econom. 6 (1979), 277-282.

2. E. J. Balder, A unified approach to several results involving integrals of multifunctions, Set-Valued Anal. 2 (1994), 63-75.

3. _ـ A unifying approach to existence of Nash equilibria, Int. J. Game Theory 24 (1995), 79-94.

4. $207-223$.

5. Incompatibility of usual conditions for equilibrium existence in continuum economies without ordered preferences, J. Econ. Theory 93 (2000), no. 1, 110-117.

6. L__ Lectures on Young measure theory and its applications in economics, Rend. Istit. Mat. Univ. Trieste 31 (2000), 1-69.

7. _ A unifying pair of Cournot-Nash equilibrium existence results, J. Econ. Theory 102 (2002), 437-470.

8. Erik J. Balder and Nicholas C. Yannelis, Equilibria in random and Bayesian games with a continuum of players., Equilibrium theory in infinite dimensional spaces, Springer-Verlag, Berlin, 1991, pp. 333-350.

9. C. Castaing and M. Valadier, Convex Analysis and Measurable Multifunctions, Lecture notes in Mathematics Vol.480, Springer-Verlag, New-York, 1977.

10. B. Cornet, M. Topuzu, and A. Yildiz, Equilibrium theory with a measure space of possibly satiated consumers, J. Math. Econom. 39 (2003), no. 3-4, 175-196.

11. M. Florenzano, General equilibrium analysis: Existence and optimality properties of equilibria, Kluwer Academic Publishers, 2003.

12. D. Gale and A. Mas-Colell, An equilibrium existence theorem for a general model without ordered preferences, J. Math. Econom. 2 (1975), 9-15.

13. M. A. Khan and N. S. Papageorgiou, On Cournot-Nash equilibria in generalized qualitative games with a continuum of agents, Nonlinear Anal. 11 (1987), 1987. 
14. __ On Cournot-Nash equilibria in generalized qualitative games with an atomless measure space of agents, Proc. Amer. Math. Soc. 100 (1987), 505-510.

15. M. A. Khan and M. Vohra, Equilibrium in abstract economies without ordered preferences and with a measure space of agents, J. Math. Econom. 13 (1984), 133-142.

16. T. Kim, K. Prikry, and N. C. Yannelis, Equilibria in abstract economies with a measure space of agents and with an infinite dimensional strategy space, J. Approx. Theory 56 (1989), 256-266.

17. V. F. Martins-da-Rocha, Equilibria in large economies with a separable Banach commodity space and non-ordered preferences, J. Math. Econom. 39 (2003), no. 8, 863-889.

18. __ Equilibria in large economies with differentiated commodities and non-ordered preferences, Econom. Theory 23 (2004), no. 3, 529-552.

19. M. Noguchi, Economies with a continuum of agents with the commodity-price pairing $\left(l_{\infty}, l_{1}\right)$, J. Math. Econom. 28 (1997), no. 3, 265-287.

20. Economies with a continuum of consumers, a continuum of suppliers and an infinitedimensional commodity space, J. Math. Econom. 27 (1997), no. 1, 1-21.

21. M. F. Sainte Beuve, On the extension of von Neumann-Aumann's theorem, J. Funct. Anal. 12 (1974), no. 1, 112-129.

22. D. Schmeidler, Competitive equilibria in markets with a continuum of traders and incomplete preferences, Econometrica 37 (1969), 578-585.

23. L. Schwartz, Radon Measures on Arbitrary Topological Spaces and Cylindrical Measures, Oxford University Press, 1973.

24. W. Shafer and H. Sonnenschein, Equilibrium in abstract economies without ordered preferences, J. Math. Econom. 2 (1975), 345-348.

25. N. C. Yannelis, Equilibria in noncooperative models of competition, J. Econ. Theory 41 (1987), 96-111.

26. _ Integration of Banach-valued correspondences, Equilibrium theory in infinite dimensional spaces, Springer-Verlag, Berlin, 1991, pp. 2-35.

Ceremade, Université Paris-IX Dauphine, Place du Marchal de Lattre de Tassigny, 75775 PARIS CEDEX 16, FRANCE

E-mail address: martins@ceremade.dauphine.fr

Cermsem, Université Paris-I Panthéon Sorbonne, 106-112 Boulevard de l'Hôpital, 75647 PARIS CEDEX 13, FRANCE

E-mail address: Mihaela.Topuzu@univ-paris1.fr 